\title{
Estacionalidad de la densidad larval del mosquito Anopheles aquasalis (Diptera: Culicidae) y otros insectos asociados a su hábitat en Sucre, Venezuela
}

\author{
Jesús Berti ${ }^{1}$, Julio González ${ }^{1}$, Edith Navarro-Bueno ${ }^{2}$, Evelin Zoppi ${ }^{3}$, Elizabeth Gordon ${ }^{3}$ \\ \& Laura Delgado ${ }^{3}$ \\ 1. Instituto de Altos Estudios "Dr. Arnoldo Gabaldon", Ministerio del Poder Popular para la Salud. Centro de \\ Investigación en Enfermedades Endémicas, Laboratorio Entomológico de Malaria, Calle Dr. Arnoldo Gabaldon, Las \\ Delicias, Maracay, Venezuela. Telef. 0243-2412846; jbertimoser@yahoo.com \\ 2. Universidad Central de Venezuela, Facultad de Agronomía, Doctorado en Ciencias, Postgrado de Entomología, \\ Maracay, Aragua, Venezuela. \\ 3. Universidad Central de Venezuela, Facultad de Ciencias, Instituto de Zoología Tropical, Ciudad Universitaria, \\ Caracas, Venezuela.
}

Recibido 07-V-2009. Corregido 20-IX-2009. Aceptado 23-X-2009.

\begin{abstract}
Larval seasonality of the mosquito Anopheles aquasalis (Diptera: Culicidae) and other insects associated to its habitat in Sucre, Venezuela. Anopheles aquasalis Curry is considered the main vector of human malaria in Northern Venezuela. A longitudinal study was carried out in the coastal areas of the Paria Peninsula, Sucre state. The larval habitats of A. aquasalis were classified as: 1- Brackish mangrove, and 2- Freshwater herbaceous swamp. Field surveys of mosquito larvae and aquatic insects were carried out in the same breeding sites over a one-year period, between January and December 1999. At each site, 30 samples of Anopheles larvae and aquatic insects were taken monthly. Simultaneously with mosquito larvae sampling, five selected variables of water were measured: conductivity, salinity, dissolved oxygen, temperature and $\mathrm{pH}$. Seasonal and temporal variations of $A$. aquasalis larvae and aquatic insects were determined in the two larval habitats. For the entire study period, the abundance of larvae was higher in the mangrove. Correspondence analysis showed a strong relation between some chemical factors of water and larval abundance. The abundance of A. aquasalis larvae in both seasons, was positively correlated with water salinity, $\mathrm{pH}$ and conductivity, and negatively and with dissolved oxygen in the dry season. The presence of larvae was positively correlated with the presence of Avicenia germinans. In the mangrove there was a positive association between larvae abundance and Scirtidae family abundance and a negative correlation between larvae abundance and monthly precipitation (Spearman), as well as a significant negative correlation between Gerridae abundance and monthly precipitation. In the herbaceous swamp, there were not significant associations between A. aquasalis larvae abundance and abundance of others aquatic insects associated to habitat. Rev. Biol. Trop. 58 (2): 777-787. Epub 2010 June 02.
\end{abstract}

Key words: seasonal and temporal variations, Anopheles aquasalis, larval habitat, aquatic insects, physicalchemical factors, malaria vectors, Venezuela.

El principal vector de la malaria humana en el oriente de Venezuela y particularmente en el estado Sucre, es el mosquito Anopheles aquasalis Curry (Zimmerman \& Berti 1994). Los problemas asociados con el control de la malaria en ese estado, han generado una serie de investigaciones y publicaciones sobre ecoepidemiología (Barrera et al. 1998, 1999, Delgado et al. 2004), la ecología de los vectores (Berti et al. 1993, 1998, 2003, 2004, Grillet et al. 1998, Grillet 2000, Grillet et al. 2002a, 2002b, Zoppi et al. 2002) y el manejo integrado 
de vectores (Zimmerman \& Berti 1994, Berti \& Zimmerman 1998, Berti et al. 2002, Berti \& González 2004, Delgado 2005).

En Venezuela, la ecología de criaderos de A. aquasalis solo ha sido estudiada en humedades del estado Sucre y por muy pocos autores (Berti et al. 1993, Berti et al. 2004, Grillet et al. 1998, Grillet 2000, Grillet et al. 2002a, Grillet et al. 2002b, Zoppi et al. 2002), que coinciden en que la especie esta fuertemente asociada con la presencia de manglares y su abundancia se correlaciona positivamente con la salinidad del agua, siendo este parámetro muy útil para predecir la distribución espacial y la variación estacional en la abundancia de la especie. En este estado, la salinidad del agua fue el único factor fisicoquímico altamente correlacionado con la abundancia de A. aquasalis; entonces la misma parece ser un buen parámetro para predecir la abundancia de esta especie; mientras que el oxígeno disuelto fue útil para predecir la presencia y abundancia de Anopheles oswaldoi (Grillet et al. 1998, Grillet 2000). Según Berti et al. (1993), la especie A. aquasalis fue más frecuente en criaderos donde los valores de salinidad fluctuaron entre 5.2 y $38.4 \mathrm{~g} / \mathrm{l}$; asimismo Grillet et al. (1998), afirman que la especie fue más frecuente y abundante principalmente en mangares con predominio del mangle $A v i$ cenia germinans y con una salinidad promedio de 27,5ppm. No obstante, la presencia de larvas de la especie ocurre en una amplia variedad de tipos de hábitat, con un amplio gradiente de salinidades que varían desde manantiales y quebradas con salinidad menor de $0.5 \mathrm{ppt}$, pantanos herbáceos con salinidades entre 0.5 y $5.1 \mathrm{ppt}$, hasta marismas salobres y manglares con salinidades entre 5.1 y $18 \mathrm{ppt}$, e incluso manglares costeros o de franja con valores de salinidad mucho mayores de 18ppt. (Berti et al. 1993, Grillet et al. 1998, Grillet 2000). Estos autores afirman que en el estado Sucre, A. aquasalis fue la especie más abundante y frecuente con respecto al gradiente ecológico y la variedad de ambientes estudiados, asimismo señalan que no se puede afirmar que presenta un comportamiento de especialista $\mathrm{u}$ oportunista, aunque la tendencia general sugiere un comportamiento de especie oportunista o generalista.

En el estado Sucre, por más de 17 años las actividades de control del vector $A$. aquasalis se han basado principalmente en la aplicación espacial de insecticidas a volumen ultra bajo en los sitios de reposo extra-domiciliario del adulto (Berti \& Zimmerman 1998); pero hasta el año 1991 el control de este vector solo se basaba en la aplicación de insecticidas residuales mediante rociamientos domiciliarios cada tres o cuatro meses (Zimmerman \& Berti 1994, Berti \& Zimmerman 1998). Estos rociamientos dentro de la casa fueron suspendidos desde 1991, debido a la elevada zoofilia, exofilia y exofagia que tenía la hembra de A. aquasalis (Zimmerman \& Berti 1994, Berti \& Zimmerman 1998). Desafortunadamente en la actualidad existe resistencia de la especie a algunos insecticidas químicos usados para su control (Molina et al. 1997), lo cual compromete seriamente el futuro éxito del programa; ambos factores obligan a ensayar nuevos métodos de control que nos permitan el mejor manejo integrado de los vectores. Actualmente el control biológico es un componente fundamental dentro de cualquier programa de manejo integrado de vectores de malaria (Berti \& Zimmerman 1998). La utilización de agentes de control biológico, tanto depredadores de larvas, como bacterias patógenas, implica un buen conocimiento sobre la ecología del vector (Zimmerman \& Berti 1994, Berti \& Zimmerman 1998).

El uso de depredadores como estrategia de control, puede combinarse con el uso periódico de bacterias, como Bacillus thuringiensis var. israelensis y B. sphaericus, para lo cual son indispensables los estudios sobre la ecología de la entomofauna asociada al hábitat larval, junto con la dinámica poblacional de larvas del vector, especialmente para conocer las fluctuaciones temporales o mensuales de las poblaciones larvarias; lo cual será de mucha utilidad para la implementación del cronograma de aplicación de agentes de control biológico. Los productos biológicos tienen la gran ventaja de ser totalmente inocuos para la fauna acuática asociada al hábitat, además de que propician la 
participación y organización de la comunidad; aspecto por el cual siempre se permitirá su utilización junto con otros agentes de control biológico como: peces autóctonos, nemátodos o insectos depredadores que puedan ser liberados en los criaderos por los miembros de la comunidad.

Debido a esto en el presente trabajo se describen fluctuaciones temporales (mensuales) de la abundancia relativa de las larvas de $A$. aquasalis y algunas familias de insectos asociadas a su hábitat (criaderos), en dos localidades de la Península de Paria: Río Chiquito Abajo (municipio Mariño) y Río de Agua (municipio Libertador). Asimismo, el estudio pretende correlacionar la abundancia relativa de las larvas de $A$. aquasalis con la abundancia de las familias de insectos y con la precipitación; con el fin de identificar depredadores como posibles agentes naturales de control biológico e intentar establecer el momento propicio, según la época del año (seca o lluviosa) para la aplicación de agentes naturales o comerciales en los criaderos de estos mosquitos vectores.

\section{MATERIALES Y MÉTODOS}

Área de estudio: La Península de Paria, está localizada en la región nororiental de Venezuela (Fig. 1), estado Sucre, entre $10^{\circ} 27^{\prime} 00^{\prime}$ ' $\mathrm{N}-10^{\circ} 42^{\prime} 32^{\prime \prime} \mathrm{N}$ y $62^{\circ} 32^{\prime} 00^{\prime}$ ' W- 63॰ $11^{\prime} 00^{\prime}$ ' W y comprende un área geográfica de $1078 \mathrm{~km}^{2}$. Al norte de la misma se encuentra el Mar Caribe y al sur el Golfo de Paria (Fig. 1). Entre las características ambientales más representativas de la región se pueden mencionar: pendientes que varían entre 0 y $5 \%$, precipitación media anual entre 1200 y $1700 \mathrm{~mm}$ y temperatura media anual entre $27^{\circ} \mathrm{C}$ y $29^{\circ} \mathrm{C}$; dependiendo de la altitud del lugar, gran parte del área permanece con suelos muy húmedos o con grandes humedales representados principalmente por: manglares costeros (estuarios), de cuenca ( $\sin$ comunicación con el mar) y de franja; pantanos herbáceos temporales o permanentes; lagunas; canales de irrigación de cultivos (cacao y hortalizas, entre otros) y canales para el manejo de hatos o fincas ganaderas, como el Hato Río de Agua. En la península de Paria la temporada

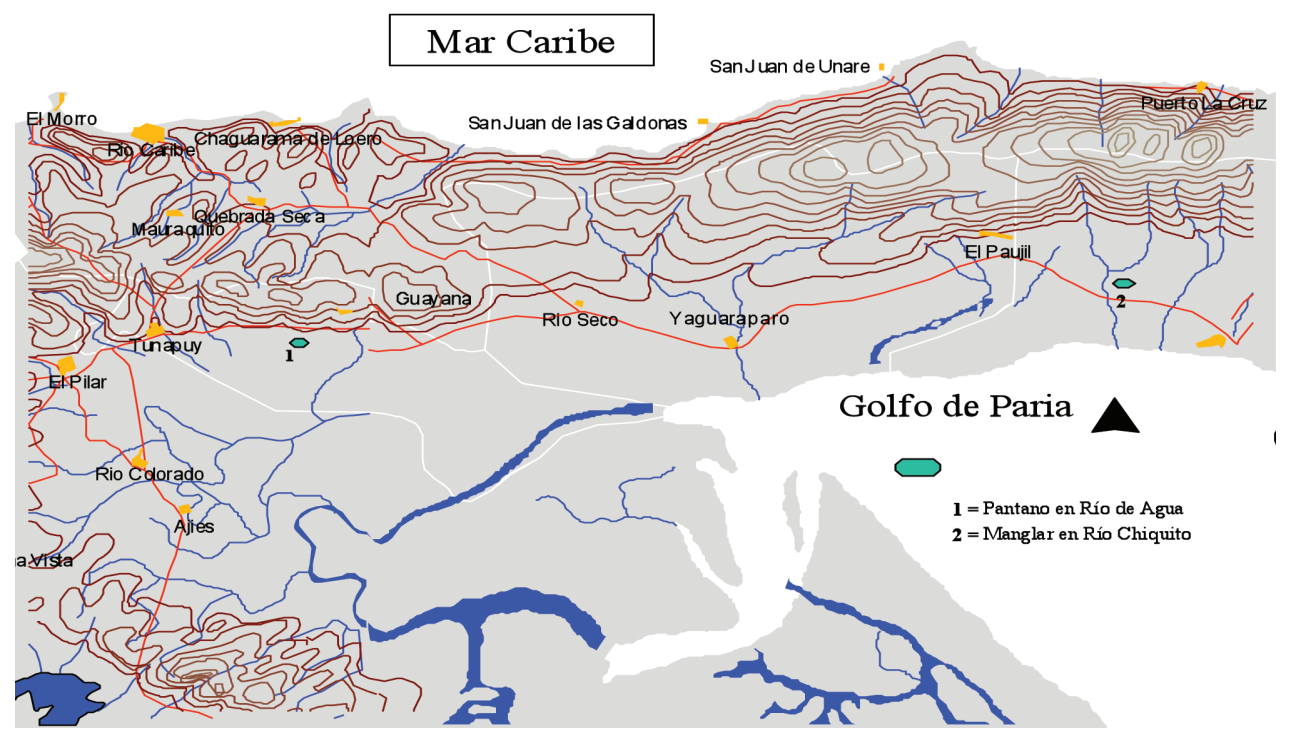

Fig. 1. Sitios de estudio en la Península de Paria, estado Sucre, Venezuela.

Fig. 1. Study sites in the Península of Paria, Sucre state, Venezuela. 
de lluvias comienza por lo general en mayo y termina a mediados de diciembre (Grillet et al. 1998, 2002a, 2002b), época del año en que comienza la sequía. Durante los meses de sequía (diciembre-abril), los criaderos de mosquitos (Anopheles) se reducen considerablemente y la mayoría llega a secarse hasta por $2 \mathrm{o}$ 3 meses. En nuestro caso, los ambientes seleccionados para el estudio permanecen con agua durante todo el año, lo que permitió realizar muestreos tanto en época seca como lluviosa y determinar la fluctuación temporal de densidad larval de $A$. aquasalis y algunas familias de insectos asociadas a su hábitat.

Tipos de hábitats seleccionados: En la localidad de Río Chiquito Abajo (10³5'29” N-62 $2^{\circ} 36^{\prime} 32^{\prime \prime} \mathrm{W}$ ), municipio Mariño se escogió un hábitat tipo "manglar" (Fig. 1), el cual fue clasificado como un manglar de cuenca. El mismo está separado del mar desde hace aproximadamente medio siglo y tanto la carretera nacional como el río Irapa, le sirven de barreras con la costa del Golfo de Paria. Aquí el régimen de inundación depende de las lluvias, la escorrentía superficial de las tierras altas adyacentes y de manantiales de aguas subterráneas (salobres). Este presenta una única especie de mangle Avicennia germinans. Esta vegetación se caracteriza por un dosel abierto, con alturas que variaron entre $20 \mathrm{~m}$ y $25 \mathrm{~m}$. Las otras especies acuáticas presentes son principalmente: Sesuvium virginicus y $S$. portulacastrum. El otro ambiente seleccionado, está situado en la finca "Hato Río de Agua" (10³4'45” N-62 58'09” W), perteneciente al municipio Libertador y ubicada al sur de la Península de Paria (Fig. 1). En el mismo existen numerosos canales de riego para el manejo de ganado, mediante la inundación permanente de pantanos herbáceos de plantas de los géneros Eleocharis y Thypha, que son usadas como alimento para los búfalos. La zona presenta intensa actividad agropecuaria (ganadería bufalina) y esta región permanece inundada prácticamente durante todo el año.

Colectas de larvas e insectos acuáticos:

En cada ambiente mencionado se tomaron mensualmente muestras de larvas con un cucharón y de insectos acuáticos con una red diseñada par tal fin. En la orilla de cada hábitat, se situó al azar una parcela $(10 \times 10 \mathrm{~m})$, la cual fue excluida del muestreo al siguiente mes, para no repetir la del mes anterior. Dentro del área seleccionada (parcela) se tomaron 30 muestras de agua, cuando las primeras 10 muestras resultaban negativas a larvas $\mathrm{u}$ otros insectos, entonces se repetía el mismo procedimiento, pero tomando 10 muestras adicionales. Las larvas de mosquitos e insectos acuáticos adultos colectados, fueron introducidos en viales con agua del criadero y se trasladaron al laboratorio donde fueron fijadas, contadas y separadas por estadios (Instares I, II, III y IV) para su posterior identificación. Cada criadero fue visitado y muestreado mensualmente durante el lapso de un año (enero a diciembre de 1999). Al momento del muestreo y mediante un equipo digital portátil (WTW, modelo 340 i/SET, Alemania) se determinó para cada parcela: la turbidez, salinidad, temperatura, conductividad, cantidad de oxígeno disuelto y $\mathrm{pH}$ del agua.

Identificación del material biológico: Para la identificación de las larvas de Anopheles se utilizó la clave de Navarro (1996). Las larvas de Anopheles del estadio IV fueron identificadas después de ser fijadas en una solución de alcohol, glicerina y ácido acético (AGA); las restantes de los otros estadios, fueron criadas hasta el estadio IV o la fase adulta, para su posterior identificación en el Laboratorio Entomológico del Instituto de Altos Estudios; los demás insectos recolectados fueron fijados con solución AGA y trasladados al mismo laboratorio para su posterior identificación. En el presente trabajo, solo se incluyen resultados correspondientes a la presencia y abundancia de insectos acuáticos de las familias de tres órdenes: Odonata, Hemiptera y Coleoptera, ya que el total de las muestras está en proceso de identificación o confirmación por especialistas de cada grupo taxonómico. No se incluyen resultados sobre la identificación de géneros y especies; ya que este trabajo solo constituye un inventario general de las familias de Odonata, 
Hemiptera y Coleoptera asociadas al hábitat de $A$. aquasalis. Posteriormente en otras publicaciones se abordarán con mayor profundidad otros importantes aspectos ecológicos sobre el hábitat de la especie, donde serán incluidas las respectivas identificaciones de los taxones género y especie.

Análisis estadístico de los datos: Las correlaciones y asociaciones estadísticas entre el tipo de hábitat, algunas variables físico-químicas del hábitat, la presencia y abundancia de larvas de $A$. aquasalis y de familias de insectos asociados, fueron determinadas mediante el método multivariado de Análisis de Correspondencia Canónica, conocido con las siglas de ACC o CANOCO (Ter Braak 1987, Ter Braak \& Smilauer 1998). También se establecieron correlaciones simples entre variables como: la precipitación mensual, la abundancia mensual de larvas de $A$. aquasalis y la abundancia mensual de familias de insectos asociados, mediante el análisis de correlación simple no paramétrica de Spearman; en ambos casos usando el programa Statistica. Estos últimos análisis incluyeron la variable precipitación solo en la localidad de Río Chiquito Abajo (10³5'29" N; $62^{\circ} 36$ '32" W); esto debido a la presencia de la estación meteorológica del INIA (Instituto Nacional de Investigaciones Agrícolas) situada a pocos kilómetros de dicha localidad. En el caso de Río de Agua (municipio Libertador), no se incluyó esta variable (precipitación) para el análisis de correlación de Spearman. En este caso solo se correlacionaron la abundancia mensual de larvas de A. aquasalis con la de las familias de insectos asociados al hábitat.

\section{RESULTADOS}

Según los resultados, los mayores índices relativos de abundancia de larvas $A$. aquasalis se presentaron en el manglar de Río Chiquito Abajo (Cuadro 1), comparados con el pantano herbáceo en el Hato Río de Agua (Cuadro 2). En el hábitat "manglar" la especie fue mucho más abundante en la época de menor pluviosidad o de sequía (febrero, marzo y abril) y menos abundante durante la temporada de lluvias, principalmente en mayo, agosto, septiembre y octubre (Cuadro 1). Este manglar está caracterizado principalmente por la presencia del mangle Avicennia germinans, que fue la especie dominante, cuya cobertura varió entre $40 \%$ y $60 \%$, la profundidad media del manglar fue de $19,30 \mathrm{~cm}(10-43 \mathrm{~cm})$, presenta sombra parcial, $\mathrm{pH}$ promedio de 8.0, altos valores de salinidad (promedio de $18.9 \mathrm{~g} / \mathrm{l}$ ) y conductividad (promedio de 29.9) y altas concentraciones de cloruros (promedio de 10.4ppm) y sulfatos (promedio de 976.6ppm). En cambio en el pantano herbáceo, ocurrió lo contrario, la mayor abundancia relativa de larvas de $A$. aquasalis se presentó durante la temporada lluviosa, principalmente en septiembre y octubre y la especie fue menos abundante en la época seca, sobre todo en abril y mayo (Cuadro 2). Este pantano se caracterizó por la ausencia total de sombra, predominio de la especie Eleocharis mutata, cuya cobertura varió entre $75 \%$ y $85 \%$, un $\mathrm{pH}$ ácido (promedio de 3.7), salinidad baja (promedio de $4.1 \mathrm{~g} / \mathrm{l}$ ) y conductividad baja (promedio de 4.3). En adición, este ambiente fue "perturbado" frecuentemente por la actividad de los búfalos que se alimentan de las plantas Eleocharis mutata, Thypha dominguensis y Thalia geniculata.

En los cuadros 1 y 2, se observa en los ambientes estudiados las fluctuaciones mensuales y estacionales de la abundancia relativa de las larvas de $A$. aquasalis y las familias pertenecientes a los órdenes Odonata, Hemiptera y Coleoptera; la mayoría de las cuales tienen hábitos depredadores y se alimentan de insectos acuáticos y larvas de mosquitos.

Los resultados del análisis de correspondencia canónica (ACC), sugieren que la abundancia relativa de larvas de $A$. aquasalis en ambas épocas (seca y lluviosa) se correlacionó positivamente y significativamente con tres variables físico-químicas: salinidad, $\mathrm{pH}$, conductividad del agua; la misma variable también se correlacionó pero en menor grado de significación con la turbidez del agua. Por otro lado, este mismo análisis, reveló que la abundancia relativa de larvas de A. aquasalis se correlacionó negativamente y significativamente con el contenido de oxígeno disuelto, pero solo en 
CUADRO 1

Abundancia mensual de larvas de A. aquasalis y de las familias de los Ordenes Hemiptera,

Odonata y Coleoptera recolectadas en el manglar de Río Chiquito Abajo

TABLE 1

Monthly abundance of A. aquasalis larvae, and Hemiptera, Odonata and Coleoptera families collected in the Rio Chiquito Abajo mangrove

\begin{tabular}{|c|c|c|c|c|c|c|c|c|c|c|c|c|}
\hline \multirow[b]{2}{*}{$\begin{array}{l}\text { Promedio mensual de } A \text {. aquasalis y } \\
\text { familias de insectos* }\end{array}$} & \multicolumn{12}{|c|}{ Mes } \\
\hline & 1 & 2 & 3 & 4 & 5 & 6 & 7 & 8 & 9 & 10 & 11 & 12 \\
\hline Larvas $A$. aquasalis & 33 & 191 & 40 & 36 & 4 & 18 & 79 & 0 & 6 & 8 & 28 & 40 \\
\hline \multicolumn{13}{|l|}{ HEMIPTERA } \\
\hline Belostomatidae & 20 & 0 & 0 & 0 & 0 & 0 & 24 & 8 & 10 & 16 & 11 & 20 \\
\hline Hydrometridae & 52 & 1 & 0 & 0 & 0 & 0 & 0 & 0 & 0 & 66 & 1 & 0 \\
\hline Notonectidae & 3 & 0 & 0 & 0 & 0 & 0 & 0 & 0 & 0 & 0 & 1 & 2 \\
\hline Naucoridae & 5 & 7 & 0 & 2 & 0 & 0 & 1 & 0 & 3 & 6 & 1 & 3 \\
\hline Corixidae & 0 & 0 & 6 & 1 & 0 & 0 & 1 & 0 & 2 & 7 & 2 & 1 \\
\hline Pleidae & 0 & 5 & 0 & 3 & 0 & 0 & 0 & 0 & 0 & 2 & 0 & 0 \\
\hline \multicolumn{13}{|l|}{ ODONATA } \\
\hline Coenagrionidae & 5 & 14 & 6 & 8 & 3 & 0 & 0 & 7 & 4 & 2 & 7 & 13 \\
\hline Libellulidae & 4 & 67 & 11 & 9 & 11 & 0 & 34 & 0 & 0 & 71 & 10 & 30 \\
\hline \multicolumn{12}{|l|}{ COLEOPTERA } & \\
\hline Hydrophilidae & 5 & 7 & 6 & 14 & 4 & 29 & 8 & 97 & 43 & 9 & 55 & 52 \\
\hline Dytiscidae & 4 & 0 & 2 & 3 & 2 & 11 & 7 & 4 & 0 & 4 & 6 & 4 \\
\hline Scirtidae & 1 & 0 & 0 & 2 & 1 & 2 & 5 & 4 & 2 & 1 & 0 & 1 \\
\hline Noteridae & 0 & 0 & 0 & 0 & 0 & 0 & 0 & 0 & 1 & 2 & 1 & 1 \\
\hline
\end{tabular}

*Número promedio de ejemplares colectados por muestra mensualmente.

la época seca. Asimismo, la presencia de larvas A. aquasalis tanto en época seca como lluviosa, está asociada positivamente con la presencia de la especie de mangle Avicennia germinans. Este análisis de correspondencia canónica, también demostró que la abundancia de larvas de $A$. aquasalis se correlacionó positivamente y significativamente con la abundancia de las familias Mesoveliidae, Pleidae, Belostomatidae, Syrphidae y Tabanidae.

Los análisis de correlación simple de Spearman correspondientes al manglar sugieren una correlación positiva y significativa (Spearman, $\mathrm{r}=0.544, \mathrm{p}<0.067$ ) entre la abundancia mensual de larvas de A. aquasalis y la abundancia mensual de la familia Scirtidae (Coleoptera). Según este análisis (Spearman), en el mismo manglar también se presentó una correlación negativa y significativa entre las siguientes variables: a) Abundancia mensual de A. aquasalis y la precipitación mensual (Spearman, $\mathrm{r}=0.553, \mathrm{p}<0.062$ ); y b) Abundancia mensual de la familia Gerridae (Hemiptera) y la precipitación mensual (Spearman, $\mathrm{r}=0.585, \mathrm{p}<0.045)$. Sin embargo, el resto de pares de variables analizadas no presentaron correlaciones estadísticamente significativas ( $p>0.062)$. Por otro lado, este análisis de correlación (Spearman) correspondiente al pantano herbáceo, sugiere la ausencia de correlaciones estadísticamente significativas entre todas las variables analizadas.

Finalmente, se presenta la lista de las familias de insectos acuáticos por cada tipo de hábitat estudiado y el número total de ejemplares recolectados por cada familia durante un año (Cuadro 3). Al respecto, hay que resaltar que las cuatro familias con mayor cantidad de 
CUADRO 2

Abundancia mensual de larvas de A. aquasalis y de las familias de los Ordenes Hemiptera, Odonata y Coleoptera recolectadas en el pantano herbáceo de Río de Agua

TABLE 2

Monthly abundance of A. aquasalis larvae and Hemiptera, Odonata y Coleoptera families collected in the Rio de Agua herbaceous swamp

\begin{tabular}{|c|c|c|c|c|c|c|c|c|c|c|c|c|}
\hline \multirow[b]{2}{*}{$\begin{array}{l}\text { Promedio mensual de } A \text {. aquasalis y } \\
\text { familias de insectos }^{1}\end{array}$} & \multicolumn{12}{|c|}{ Mes } \\
\hline & 1 & 2 & 3 & 4 & 5 & 6 & 7 & 8 & 9 & 10 & 11 & 12 \\
\hline Larvas $A$. aquasalis & 56 & 22 & 19 & 13 & 0 & 8 & 4 & 28 & 34 & 77 & 30 & 10 \\
\hline Belostomatidae & 1 & 2 & 5 & 2 & 0 & 1 & 3 & 1 & 1 & 0 & 2 & 1 \\
\hline Hydrometridae & 1 & 0 & 0 & 0 & 0 & 0 & 0 & 0 & 0 & 0 & 1 & 0 \\
\hline Notonectidae & 1 & 1 & 0 & 0 & 0 & 0 & 0 & 0 & 0 & 0 & 0 & 0 \\
\hline Naucoridae & 0 & 4 & 0 & 2 & 0 & 0 & 1 & 0 & 1 & 1 & 0 & 0 \\
\hline Gerridae & 3 & 0 & 0 & 3 & 0 & 0 & 0 & 0 & 0 & 0 & 0 & 0 \\
\hline Veliidae & 4 & 4 & 2 & 0 & 0 & 0 & 11 & 0 & 0 & 0 & 0 & 6 \\
\hline Corixidae & 0 & 0 & 0 & 3 & 0 & 0 & 0 & 0 & 0 & 2 & 0 & 0 \\
\hline Pleidae & 35 & 51 & 22 & 18 & 3 & 9 & 0 & 7 & 7 & 2 & 41 & 10 \\
\hline Coenagrionidae & 16 & 11 & 6 & 5 & 11 & 16 & 14 & 4 & 14 & 34 & 11 & 11 \\
\hline Libellulidae & 65 & 7 & 6 & 11 & 46 & 15 & 24 & 21 & 40 & 9 & 18 & 77 \\
\hline Aeshnidae & 0 & 1 & 0 & 0 & 0 & 1 & 1 & 1 & 0 & 1 & 1 & 0 \\
\hline Hydrophilidae & 1 & 0 & 8 & 2 & 1 & 2 & 5 & 4 & 2 & 1 & 0 & 1 \\
\hline Dytiscidae & 21 & 34 & 12 & 10 & 13 & 12 & 5 & 31 & 14 & 0 & 0 & 1 \\
\hline Scirtidae & 0 & 1 & 0 & 0 & 0 & 1 & 1 & 2 & 0 & 1 & 1 & 0 \\
\hline Noteridae & 0 & 0 & 0 & 0 & 0 & 3 & 5 & 1 & 10 & 0 & 1 & 0 \\
\hline
\end{tabular}

* Número promedio de ejemplares colectados por muestra mensualmente.

ejemplares recolectados durante el año fueron: Hydrophilidae, Pleidae, Libellulidae y Dytiscidae respectivamente. La familia Hydrophilidae (Cuadros 1, 2, 3) fue muy abundante sobre todo en el manglar de Río Chiquito. La familia Pleidae fue la más abundante en el pantano herbáceo, sobre todo en época de sequía, mientras que en el manglar fue muy escasa (Cuadros 1, 2, 3); la familia Libellulidae, fue muy abundante en ambos ambientes y aparece con mucha frecuencia, tanto en época de sequía como de lluvias (Cuadros 1, 2, 3) y la familia Dytiscidae fue mucho más frecuente y abundante en el pantano que en el manglar (Cuadros 1, 2, 3).

\section{DISCUSIÓN}

Según los resultados del análisis de correspondencia canónica, la presencia de larvas $A$. aquasalis tanto en época seca como en época lluviosa, está asociada positivamente con la presencia de la especie de mangle negro ( $A$. germinans). Este resultado coincide con los de Grillet et al. (1998) y según los mismos, la presencia de larvas de la especie en ambas épocas del año se correlacionó positivamente con el hábitat manglar y con la presencia del mangle negro. Con relación a los parámetros físico-químicos, el análisis de correspondencia canónica sugiere que la abundancia de larvas de $A$. aquasalis se correlacionó positivamente y significativamente con la salinidad, la conductividad y el $\mathrm{pH}$ del agua; resultados similares fueron señalados en otras regiones del estado Sucre por Grillet et al. (1998), Grillet (2000) y Berti et al. (2004), pero solo con respecto a la salinidad y conductividad del agua. Se reporta por primera vez: una correlación positiva entre 
CUADRO 3

Número total de ejemplares de las familias de insectos acuáticos recolectados en el manglar (Río Chiquito Abajo) y el pantano herbáceo (Hato Río de Agua)

TABLE 3

Total number of aquatic insects collected in the mangrove (Rio Chiquito Abajo) and the herbaceous swamp (Hato Río de Agua)

Familia de insecto *

1- Belostomatidae

2- Hydrometridae

3- Notonectidae

4- Mesoveliidae

5- Naucoridae

6- Gerridae

7- Veliidae

8- Nepidae

9- Corixidae

10- Pleidae

11- Coenagrionidae

12- Calopterygidae

13- Libellulidae

14- Aeshnidae

15- Gomphidae

16- Hydrophilidae

17- Dytiscidae

18- Noteridae

19- Scirtidae
Pantano herbáceo del Hato Río de Agua **

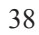

10

13

2

15

5

11

1

21

234

70

0

115

21

0

29

122

0

5
Manglar de Río Chiquito Abajo **

28

1

8

1

30

11

1

0

41

11

26

7

131

0

1

394

21

9

14

*Número que identifica cada familia del orden respectivo: Orden Hemiptera=1-10; Orden Odonata=11-15; y Orden Coleoptera $=16-19$.

**Número total de ejemplares colectados de cada familia.

el $\mathrm{pH}$ y la abundancia de larvas de $A$. aquasalis y una correlación negativa y significativa entre el contenido de oxígeno disuelto y la abundancia de larvas de A. aquasalis, pero solo en la época seca. Sin embargo, Grillet (2000) no encontró correlación entre el oxígeno y la abundancia de larvas de A. aquasalis, pero señaló que la presencia y la abundancia de larvas de Anopheles oswaldoi (Peryassú) si está fuertemente correlacionada con el oxígeno disuelto (Grillet 2000).

El análisis de correspondencia canónica, también demostró que la abundancia de larvas de $A$. aquasalis se correlacionó positiva y significativamente con la abundancia de las familias Mesoveliidae, Pleidae, Belostomatidae, Syrphidae y Tabanidae; de las cuales solamente las tres de Hemiptera: Mesoveliidae, Pleidae y Belostomatidae tienen hábitos de depredación; sin embargo, los resultados de este análisis no apoyan la suposición de que sean depredadoras de larvas, ya que de existir correlaciones negativas se esperaría que al aumentar las densidades del depredador debería disminuir la abundancia de las larvas, pero ese no fue el caso y las correlaciones fueron positivas, lo que implica que estas familias no serían muy efectivas como controladores biológicos de A. aquasalis en el estado Sucre. En ningún trabajo previo se señalan correlaciones 
negativas entre familias o géneros de insectos depredadores y la abundancia de A. aquasalis. En el manglar se encontró correlación positiva entre la abundancia mensual de larvas de A. aquasalis y la abundancia mensual de la familia Scirtidae (Coleoptera); este resultado tampoco tiene mucha relevancia, debido a que esta familia no presenta géneros con hábitos de depredación, y por el contrario generalmente estos son herbívoros y comedores de detritus, materia vegetal en descomposición, etc (Roldan-Pérez 1988). La inexistencia de otras investigaciones sobre alguna de estas familias con relación a su potencial como controladores biológicos de mosquitos, unido al hecho de que hasta el presente, en el país todavía no se ha implementado un programa de liberaciones masivas y periódicas de depredadores naturales de larvas, le confieren mucha relevancia a los resultados del presente estudio.

Según Zoppi et al. (2002), en un pantano herbáceo con predominio de Eleocharis muta$t a$, se encontró una correlación negativa entre la abundancia del copépodo Mesocyclops meridianus y la de las larvas de $A$. aquasalis; siendo esta la primera y única cita al respecto en el país y con relación al papel de estos crustáceos como agentes potenciales de control biológico de larvas. No obstante, la biología poblacional de vectores de la malaria y de artrópodos asociados al hábitat, ha sido estudiada por algunos autores (Berti et al. 2003, Grillet et al. 1998, Grillet 2000, Grillet et al. 2002a, 2002b, Zoppi et al. 2002). En estos trabajos se señalan resultados sobre las fluctuaciones estacionales y los factores ambientales que afectan la presencia, ausencia y abundancia de numerosas familias de los órdenes Coleoptera, Hemiptera, Odonata, Diptera y Ephemeroptera, todas asociadas al hábitat de algunas especies de Anopheles. Sin embargo, en todos ellos, los resultados sobre la abundancia de estas familias, a diferencia del presente estudio, solo fueron correlacionados con factores físico-químicos del hábitat. En ese sentido, Grillet et al. (2002a, 2002b) señalaron la presencia de correlaciones positivas y altamente significativas entre la abundancia de las familias Corixidae y Notonectidae con la profundidad del criadero y la temperatura del agua; asimismo informan de correlaciones positivas y significativas de las familias Libellulidae, Coenagrionidae y Mesoveliidae con la profundidad del criadero y también de la familia Veliidae con la profundidad del criadero, el oxígeno disuelto, la conductividad y la temperatura del agua; sin embargo, estos autores no informan sobre la existencia de otro tipo de correlación entre las múltiples variables del hábitat que fueron estudiadas y la abundancia de las especies de Anopheles (Grillet et al. 2002a, Grillet et al. 2002b).

En humedales de la Península de Paria, muy pocas familias de insectos son dominantes al iniciarse el periodo lluvioso, entre ellas está principalmente la familia Culicidae, incluyendo las especies de Anopheles (Grillet et al. 2002a). Es conocido que en el estado Sucre, el principal factor ambiental que regula la emergencia y abundancia de adultos de $A$. aquasalis, es la presencia o ausencia de sus principales criaderos que durante la temporada lluviosa son pantanos herbáceos tanto estacionales o temporales como permanentes (Zimmerman \& Berti 1994); asimismo, se sabe que la abundancia de estos criaderos principales está directamente relacionada con el período lluvioso que comprende los meses entre mayo y diciembre (Berti et al. 1993, Grillet et al. 1998, Grillet et al. 2002a, 2002b, Berti et al. 2004). en el presente estudio, A. aquasalis fue más abundante en el manglar durante la época de sequía y en el pantano lo fue en la temporada lluviosa; estos resultados nos obligan a planificar el programa de manejo integrado del vector tomando en cuenta estas diferencias. En el manejo integrado del vector (Berti \& Zimmerman 1998, Berti \& González 2004, delgado 2005), se recomienda efectuar la primera aplicación de Bacillus thuringiensis var. israelensis o Bacillus sphaericus durante el mes de abril o al inicio de mayo; es decir, justo antes del inicio de las lluvias y cuando todavía las densidades larvales son bajas. Esta estrategia, entonces es muy recomendable para la gran mayoría de criaderos en época lluviosa, así como para pantanos herbáceos, charcos, lagunas, etc. De 
esta manera, se reduciría considerablemente la proporción de adultos que emergen para dispersarse y colonizar otros criaderos recién formados al inicio de la nueva temporada lluviosa, y que aún están libres de depredadores naturales. En consecuencia, si mediante esta primera aplicación de la bacteria, se logra contener el incremento poblacional de la especie, típico de las primeras precipitaciones (Berti et al. 1993, Grillet et al. 1998, Berti et al. 2004), entonces los depredadores naturales autóctonos podrían ser liberados masivamente mediante una activa participación comunitaria; para así ejercer una mayor acción reguladora sobre las poblaciones larvales del vector (Grillet et al. 1998). En el caso de marismas y manglares salobres, la primera aplicación de la bacteria, se debería realizar al final de la temporada lluviosa o al inicio de la época seca, para contener el incremento poblacional de la especie, que se observa en los meses de sequía; entonces posteriormente los depredadores naturales autóctonos también podrían ser liberados masivamente mediante la activa participación ciudadana; todo lo anterior nos permitirá alargar el tiempo previsto para efectuar una segunda aplicación de la bacteria B. sphaericus.

\section{AGRADECIMIENTOS}

Gracias a Melfran Herrera y Frances Osborn por su valiosa colaboración en el trabajo de campo. A Lettty González por su apoyo logístico y Alfredo Gutiérrez por asesorar en el análisis estadístico de los datos.

\section{RESUMEN}

Anopheles aquasalis es considerado como el principal vector de malaria humana en el norte de Venezuela. El estudio longitudinal se llevó a cabo en áreas costeras de la Península de Paria, estado Sucre. El hábitat de las larvas de $A$. aquasalis fue clasificado como: manglar salobre y pantano herbáceo. Muestreos para recolectar larvas de mosquitos e insectos asociados se realizaron mensualmente en ambos criaderos desde enero hasta diciembre de 1999 (30 muestras). Simultáneamente se midieron cinco variables seleccionadas del agua: conductividad, salinidad, oxígeno disuelto, temperatura y $\mathrm{pH}$. En ambos criaderos de determinaron las variaciones estacionales y temporales de las larvas de A. aquasalis e insectos acuáticos. Para el período de estudio, la abundancia de larvas fue mayor en el manglar. El análisis de correspondencia mostró una fuerte relación entre algunos factores químicos del agua y la abundancia de las larvas. La abundancia de las larvas de A. aquasalis en ambas épocas, se correlacionó positivamente con la salinidad del agua, $\mathrm{pH}$ y conductividad, y negativamente con el oxígeno disuelto, en la estación seca. La presencia de larvas se correlacionó positivamente con la presencia de Avicennia germinans. En el manglar existió una asociación positiva entre la abundancia de larvas y la abundancia de la familia Scirtidae y una correlación negativa entre la abundancia de larvas y la precipitación mensual (Spearman), así como una correlación negativa significativa entre la abundancia de Gerridae y la precipitación mensual. En el pantano herbáceo, no había asociaciones significativas entre la abundancia de las larvas de A. aquasalis y la abundancia de otros insectos acuáticos asociados al hábitat.

Palabras clave: fluctuación temporal y estacional, Anopheles aquasalis, hábitat larval, insectos acuáticos, parámetros físico-químicos, vectores de malaria, Venezuela.

\section{REFERENCIAS}

Barrera, R., M. Grillet, Y. Rangel, J. Berti \& A. Aché. 1998. Estudio Eco-epidemiológico de la reintroducción de la malaria en el nororiente de Venezuela, mediante sistemas de información geográfica y sensores remotos. Bol. Dir. Malariol. San. Amb. 38: 14-30.

Barrera, R., M. Grillet, Y. Rangel, J. Berti \& A. Aché. 1999. Temporal and spatial patterns of malaria reinfection in northeastern of Venezuela. Am. J. Trop. Med. Hyg. 61: 784-790.

Berti, J., R. Zimmerman \& J. Amarista. 1993. Spatial and temporal distribution of anopheline larvae in two malarious areas of Sucre State, Venezuela. Mem. Inst. Oswaldo Cruz. 88: 353-362.

Berti, J. \& R. Zimmerman. 1998. Métodos para el control integrado de los vectores de la malaria en Venezuela. Bol. Dir. Malariol. San. Amb. 38: 123-136.

Berti, J., J. González, C. Vanegas, H. Guzmán \& J. Amarista. 1998. Fluctuaciones estacionales de las densidades larvarias de Anopheles pseudopunctipennis en ríos de Santa Fe, estado Sucre, Venezuela. Bol. Entomol. Venez. 13: 1-15.

Berti, J., X. Ramírez, M. Herrera. \& J. González. 2002. Evaluación de la efectividad Bacillus sphaericus contra de larvas de Anopheles aquasalis en criaderos naturales del estado Sucre. Entomotropica 17: 1-5. 
Berti, J., E. Navarro-Bueno \& J. González. 2003. Lista de géneros de Coleoptera asociados al hábitat larvario de Anopheles aquasalis (Diptera: Culicidae) en humedales de la Península de Paria, Venezuela. Bol. Malariol. Sal. Amb. 43: 31-36.

Berti, J. \& J. González. 2004. Evaluación de la efectividad y persistencia de una nueva formulación de Bacillus sphaericus contra larvas de Anopheles aquasalis en humedales de la Península de Paria, estado Sucre, Venezuela. Bol. Malariol. Sal. Amb. 44: 21-28.

Berti, J., A. Gutiérrez \& R. Zimmerman. 2004. Relaciones entre tipos de hábitat, algunas variables químicas y la presencia de larvas de Anopheles aquasalis Curry y Anopheles pseudopunctipennis Theobald en un área costera del estado Sucre, Venezuela. Entomotropica 19: 79-84.

Delgado, L., S. Ramos, E. Zoppi, E. Gordon, E. Montiel \& J. Berti. 2004. Nuevas perspectivas para manejo de la Salud Pública: Caso particular de la malaria en el estado Sucre. Rev. Fac. Ing. UCV 19: 51-61.

Delgado, N. 2005. Factores que afectan la eficacia y persistencia de Bacillus thuringiensis var. israelensis sobre Anopheles aquasalis Curry (Diptera: Culicidae), vector de malaria en Venezuela. Entomotropica 20: $213-233$.

Grillet, M., H. Montañez \& J. Berti. 1998. Estudio Biosistemático y ecológico de Anopheles aquasalis y sus implicaciones para el control de malaria en el estado Sucre. II. Ecología de criaderos. Bol. Dir. Malariol. San. Amb. 38: 38-46.

Grillet, M.A. 2000. Factors associated with distribution of Anopheles aquasalis and Anopheles oswaldoi (Diptera: Culicidae) in a malarious area, northeastern Venezuela. J. Med. Entomol. 37: 231-238.

Grillet, M., P. Legendre \& D. Borcard. 2002a. Community structure of Neotropical wetland insects in Northern
Venezuela. I. Temporal and environmental factors. Arch. Hydrobiol. 155: 413-436.

Grillet, M., P. Legendre \& D. Borcard. 2002b. Community structure of Neotropical wetland insects in Northern Venezuela. II. Habitat type and environmental factors. Arch. Hydrobiol. 155: 437-453.

Molina, D., F. Saume, J. Bisset, O. Hidalgo, M. Castillo, W. Anaya, O. Salas, J. González \& H. Barazarte. 1997. Establecimiento de la línea de susceptibilidad a insecticidas en la fase adulta de Anopheles spp. (Diptera: Culicidae) de Venezuela. Bol. Dir. Malariol. San. Amb. 37: 55-69.

Navarro, J.C. 1996. Actualización de la tribu Anophelini de Venezuela, con una nueva clave para la identificación de larvas. Bol. Dir. Malariol. San. Amb. 36: 25-43.

Roldán-Pérez, G. 1988. Guía para el estudio de macroinvertebrados acuáticos del Departamento de Antioquia. Presencia, Bogotá, Colombia.

Ter Braak, C.J. 1987. The analysis of vegetation: environment relationships by canonical correspondence analysis. Vegetario 69: 69-77.

Ter Braak, C. \& P. Smilauer. 1998. CANOCO reference manual and user guide to Canoco for Windows. Software for canonical community ordination. Microcomputer Power. Ithaca, Nueva York, EEUU.

Zimmerman, R. \& J. Berti. 1994. The importance of integral control of malaria for the preservation of wetlands in Latin America, p. 797-803. In W. Witson (ed.) Global Wetlands: Old World and New. Elsevier Science, Ohio State University, Ohio, EEUU.

Zoppi, E., E. Gordon, L. Delgado, J. Berti \& S. Ramos. 2002. Association of cyclopoid copepods with the habitat of the malaria vector Anopheles aquasalis in the Península of Paria, Venezuela. J. Amer. Mosq. Contr. Assoc. 18: 47-51. 
